\title{
A Characterization of Quasi-Homogeneous Purely Elliptic Complete Intersection Singularities
}

\author{
HENRIK VOSEGAARD \\ Department of Mathematics, Aarhus University, Ny Munkegade, DK-8000 Aarhus C, \\ Denmark; e-mail: vosegaard@imf.au.dk
}

(Received: 9 July 1999; accepted in final form: 29 July 1999)

\begin{abstract}
It is well-known that quasi-homogeneity is characterized by equality of the Milnor and Tjurina numbers for isolated complex analytic hypersurface singularities and for certain low-dimensional singularities. In this paper we prove that this characterization extends to isolated purely elliptic complete intersection singularities, with bounds on neither the embedding codimension nor the dimension of the singularity.
\end{abstract}

Mathematical Subject Classifications (2000). 32S05, 32S35, 14M10, 14E15.

Key words. quasi-homogeneous singularities; purely elliptic singularities; equality of Milnor and Tjurina numbers; mixed Hodge theory of singularities.

\section{Introduction}

The famous theorem of K. Saito [Sa] from 1971 states that an isolated hypersurface singularity is quasi-homogeneous (i.e. has a good $\mathbb{C}^{*}$-action) if and only if the Milnor number equals the Tjurina number. The question of characterizing quasihomogeneity for other types of singularities has subsequently occupied many authors (see, e.g., [Wal], [G2], [Wahl2], [HM], [XY], [V], to mention but a few), and Saito's result has been extended to isolated complete intersection singularities of dimension 1 by G.-M. Greud, B. Martin and G. Pfister [GMP] (see also [G2, 2.6(3)]) and dimension 2 by $\mathrm{J}$. Wahl [Wahl2] using rather different techniques.

In this paper we shall focus on higher-dimensional isolated complete intersection singularities. The Milnor number and Tjurina number are defined for such singularities, and it is natural to ask if Saito's result generalizes? The main theorem below gives an affirmative answer for purely elliptic singularities (1.4) which form the border line between rational and 'general' singularities (they are Du Bois but non-rational or, in different terms, log-canonical but non-canonical). In contrast with earlier results it is interesting to note that within the class of purely elliptic singularities there are no bounds on dimension and embedding codimension.

Throughout the paper a singularity will mean a germ $(X, x)$ of a $\mathbb{C}$-analytic space. For an isolated complete intersection singularity (ICIS for short) one defines the Milnor number $\mu=\mu(X, x)$ as the number of spheres appearing in the vanishing 
cohomology, and the Tjurina number $\tau=\tau(X, x)$ is the $\mathbb{C}$-dimension of the space $T_{X, x}^{1}$ of first-order deformations of $(X, x)$.See [L] for details.

MAIN THEOREM. Let $(X, x)$ be a purely elliptic ICIS of dimension $n \geqslant 2$. Then $(X, x)$ is quasi-homogeneous if and only if $\mu=\tau$.

The 'only if'-part is due to Greuel [G1, Satz 0.3] and holds for any ICIS of positive dimension.

To find sufficient conditions for a purely elliptic ICIS $(X, x)$ to be quasi-homogeneous we start in Section 1 with a discussion on the Hodge type $(0, s)$ of $(X, x), 0 \leqslant s \leqslant n-1$, as defined by S. Ishii [I]. This invariant may be defined from the mixed Hodge theory on the $(n-1)$-cohomology of the link of $(X, x)$ and measures the 'purity' of a certain component of the Hodge-graduation. It will be clear (1.9) that Hodge type $(0, n-1)$ is a necessary condition for quasihomogeneity, but it is not quite sufficient when $n \geqslant 3$ (1.11).

E. Looijenga and J. Steenbrink gave in [LS] a formula expressing for any ICIS $(X, x)$ with $n \geqslant 2$ the difference $\mu-\tau$ as a sum of non-negative terms computed from the Hodge theory of the link and cohomological data of a choice of resolution. We extract from these data a non-negative analytic invariant $\chi=\chi(X, x)$ satisfying $\mu-\tau \geqslant \chi$. When $(X, x)$ is purely elliptic the only possible values of $\chi$ are 0 and 1 , and we prove that $\chi=0$ precisely when $(X, x)$ is quasi-homogeneous (Thm 2.6). This result is sharper than (and clearly implies) the 'if'-part of Main Theorem since the difference $\mu-\tau$ generally is much greater than $\chi$.

The proof that $\chi=0$ implies quasi-homogeneity owes much to the ideas and techniques in the papers [I] and [Wahl2] by S. Ishii and J. Wahl. For any good resolution $(Y, D) \rightarrow(X, x)$ of a purely elliptic ICIS $(X, x)$ satisfying $\chi=0$ there is a distinguished 'essential' exceptional irreducible component $D_{J} \leqslant D$. We produce a vector field (i.e. a derivation) $\tilde{\xi}$ on $(X, x)$ that lifts to a meromorphic vector field on $Y$ with a well-described logarithmic vanishing along $D_{J}$, whose action on the Zariski tangent space is non-nilpotent. By a theorem of Scheia and Wiebe [SW] this ensures quasi-homogeneity except in a more delicate special case which is treated separately.

\section{Purely Elliptic Singularities}

1.1. Let $(X, x)$ be a normal isolated singularity of dimension $n \geqslant 2$, and let $(Y, D) \stackrel{\pi}{\rightarrow}(X, x)$ be a good resolution, i.e. $Y$ is a non-singular space, $D=\pi^{-1}(x)_{\text {red }}$ is a divisor with normal crossings, and $\pi$ induces an isomorphism of $Y \backslash D$ and $X \backslash\{x\}$. We will assume that each irreducible component of $D$ is non-singular.

Choose representatives $X, Y$ such that $X$ is a good contractible Stein representative of the singularity $(X, x)$ and $Y=\pi^{-1}(X)$. Put $U=X \backslash\{x\}$ (which we identify via $\pi$ with $Y \backslash D)$. Then $U$ is homotopy equivalent to the link of $(X, x)$. Let $\Omega_{Y}^{p}$ (resp. $\Omega_{U}^{p}$ ) denote the sheaf of holomorphic $p$-forms on $Y$ (resp. $U$ ). 
Recall that the singularity $(X, x)$ is rational if $H^{i}\left(\mathcal{O}_{Y}\right)=0$ for $i>0$, while $(X, x)$ is $D u$ Bois $[\mathrm{St} 1,3.6]$ if the natural map $H^{i}\left(\mathcal{O}_{Y}\right) \stackrel{q_{i}}{\rightarrow} H^{i}\left(\mathcal{O}_{D}\right)$ is an isomorphism for $i>0$. By [St1, Lemma 2.14] $q_{i}$ is surjective for all $i$, hence rational singularities are also Du Bois. Moreover, $(X, x)$ is Du Bois if and only if $H^{i}\left(\mathcal{O}_{Y}(-D)\right)=0$ for $i>0$.

1.2. Now assume that $(X, x)$ is Gorenstein. By Serre duality and the theorem of Grauert and Riemenschneider $H_{D}^{i}\left(\mathcal{O}_{Y}\right)=0$ for $i \neq n$, so $H^{i}\left(\mathcal{O}_{Y}\right)=H^{i}\left(\mathcal{O}_{U}\right)=0$ for $i \neq 0, n-1$. Therefore, $(X, x)$ is rational (resp. Du Bois) if and only if $H^{n-1}\left(\mathcal{O}_{Y}\right)=0\left(\right.$ resp. $\left.H^{n-1}\left(\mathcal{O}_{Y}(-D)\right)=0\right)$. The composite

$$
H_{D}^{n-1}\left(\mathcal{O}_{Y}(-D)\right) \hookrightarrow H^{n-1}\left(\mathcal{O}_{Y}(-D)\right) \hookrightarrow H^{n-1}\left(\mathcal{O}_{Y}\right)
$$

factors through $H_{D}^{n-1}\left(\mathcal{O}_{Y}\right)=0$, hence $H_{D}^{n-1}\left(\mathcal{O}_{Y}(-D)\right)=0$, and dually, $H^{1}\left(\Omega_{Y}^{n}(D)\right)=0$. It is now immediate that $(X, x)$ is Du Bois if and only if $H^{0}\left(\Omega_{Y}^{n}(D)\right) \rightarrow H^{0}\left(\Omega_{U}^{n}\right)$ is surjective. Similarly, $(X, x)$ is rational if and only if $H^{0}\left(\Omega_{Y}^{n}\right) \rightarrow H^{0}\left(\Omega_{U}^{n}\right)$ is surjective.

1.3. Du Bois and rational singularities are also characterized in terms of their discrepancies. Write $K_{Y}=\pi^{*} K_{X}+D_{I}-D_{J}$, where $D_{I}$ and $D_{J}$ are effective exceptional divisors having no irreducible components in common. (The notation is due to Ishii [I, Def. 3.3] who calls $D_{J}$ the essential divisor of $\pi$.) A choice $\omega \in H^{0}\left(\Omega_{U}^{n}\right)$ of generator (as a cyclic $H^{0}\left(\mathcal{O}_{X}\right)$-module) gives rise to an isomorphism

$$
\omega: \mathcal{O}_{Y}\left(D_{I}-D_{J}\right) \stackrel{\cong}{\rightrightarrows} \Omega_{Y}^{n} .
$$

Using Serre-duality and the theorem of Grauert and Riemenschneider it is not hard to show that the natural map $H^{n-1}\left(\mathcal{O}_{Y}\right) \rightarrow H^{n-1}\left(\mathcal{O}_{D_{J}}\right)$ is an isomorphism.

By the above, $(X, x)$ is rational (resp. Du Bois) if and only if $D_{J}=0$ (resp. $D_{J} \leqslant D$ ). Note that the condition $D_{J}=0$ also defines canonical singularities, while $D_{J} \leqslant D$ defines log-canonical singularities.

DEFINITION 1.4 [Wat, 3.16]. A normal Gorenstein singularity $(X, x)$ is called purely elliptic if it is Du Bois but not rational (equivalently, the essential divisor $D_{J}$ is reduced and non-trivial).

From the discussions in 1.1 and 1.3 we see that for a Du Bois singularity there are natural isomorphisms

$$
H^{n-1}\left(\mathcal{O}_{Y}\right) \stackrel{\cong}{\rightrightarrows} H^{n-1}\left(\mathcal{O}_{D}\right) \stackrel{\cong}{\rightrightarrows} H^{n-1}\left(\mathcal{O}_{D_{J}}\right)
$$

These spaces are trivial in the rational case and one-dimensional as vector spaces in the purely elliptic case.

1.5. Now recall that the cohomology groups of $U$ (for any normal singularity $(X, x)$ ) carry canonical mixed Hodge structures [St1, Sect. 1]. We let $h^{i, j}$ denote the $(i, j)^{\prime}$ th 
mixed Hodge number of $H^{n-1}(U, \mathbb{C})$, i.e.

$$
h^{i, j}=\operatorname{dim} \operatorname{Gr}_{F}^{i} \operatorname{Gr}_{i+j}^{W} H^{n-1}(U, \mathbb{C}) .
$$

By the semipurity theorem [St1, Cor. 1.12], $h^{i, j}=0$ unless $i, j \geqslant 0$ and $i+j \leqslant n-1$.

From [LS] we obtain isomorphisms

$$
\operatorname{Gr}_{F}^{p} H^{p+q}(U, \mathbb{C}) \stackrel{\cong}{\rightrightarrows} H^{q}\left(\Omega_{Y}^{p}(\log D) \otimes \mathcal{O}_{D}\right),
$$

where $\Omega_{Y}^{p}(\log D)$ is the sheaf of holomorphic $p$-forms on $Y$ with logarithmic poles along $D$. In particular,

$$
\mathrm{Gr}_{F}^{0} H^{n-1}(U, \mathbb{C}) \stackrel{\cong}{\rightrightarrows} H^{n-1}\left(\mathcal{O}_{D}\right) .
$$

When $(X, x)$ is purely elliptic, it follows from the remark after Definition 1.4 that $h^{n-1}\left(\mathcal{O}_{D}\right)=1$. Consequently, exactly one of the numbers $h^{0, i}$ is equal to 1 , while the rest vanish.

DEFINITION 1.6 [I, Sect. 4]. A purely elliptic singularity $(X, x)$ is of Hodge type $(0, s)$, if $h^{0, s}=1$.

Clearly, $0 \leqslant s \leqslant n-1$ when $n$ is the dimension of $(X, x)$ and $(0, s)$ its Hodge type.

Note that the Hodge type $(0, s)$ is also characterized by the demand that $H^{n-1}\left(\mathcal{O}_{D_{J}}\right)=\operatorname{Gr}_{F}^{0} H^{n-1}\left(D_{J}, \mathbb{C}\right)$ is pure of weight $s$ (by (1.4.1)), hence our definition of Hodge type is equivalent to the one given by Ishii (see [I, Def. 4.1] and the following remark).

We will give two characterizations of $(X, x)$ being of Hodge type $(0, n-1)$. The first is essentially proved in [I, Thm. 4.3] and the proof is taken from there.

LEMMA 1.7. Assume that $(X, x)$ is purely elliptic of dimension $n$. Then $(X, x)$ is of Hodge type $(0, n-1)$ if and only if the essential divisor $D_{J}$ is irreducible.

Proof. If $D_{J}$ is irreducible, then $D_{J}$ is non-singular and the mixed Hodge structure on $H^{n-1}\left(D_{J}, \mathbb{C}\right)$ is pure of weight $n-1$. By the remark following Definition 1.6, $(X, x)$ must be of Hodge type $(0, n-1)$.

Conversely, assume $(X, x)$ is of Hodge type $(0, n-1)$. If $D_{J}=D_{1}+D_{2}$ for non-trivial effective divisors $D_{1}, D_{2}$, then by Mayer-Vietoris,

$$
\begin{gathered}
\operatorname{Gr}_{F}^{0} \operatorname{Gr}_{n-1}^{W} H^{n-2}\left(D_{1} \cap D_{2}, \mathbb{C}\right) \rightarrow \operatorname{Gr}_{F}^{0} \operatorname{Gr}_{n-1}^{W} H^{n-1}\left(D_{J}, \mathbb{C}\right) \rightarrow \\
\operatorname{Gr}_{F}^{0} \operatorname{Gr}_{n-1}^{W} H^{n-1}\left(D_{1}, \mathbb{C}\right) \oplus \operatorname{Gr}_{F}^{0} \operatorname{Gr}_{n-1}^{W} H^{n-1}\left(D_{2}, \mathbb{C}\right)
\end{gathered}
$$

is exact. Now,

$$
\operatorname{Gr}_{F}^{0} H^{n-1}\left(D_{i}, \mathbb{C}\right)=H^{n-1}\left(\mathcal{O}_{D_{i}}\right)=0, \quad i=1,2
$$

by [I, Cor 3.9], while $\operatorname{Gr}_{n-1}^{W} H^{n-2}\left(D_{1} \cap D_{2}, \mathbb{C}\right)=0$ since $D_{1} \cap D_{2}$ is complete of 
dimension $<n-1$. Thus, $\operatorname{Gr}_{F}^{0} \mathrm{Gr}_{n-1}^{W} H^{n-1}\left(D_{J}, \mathbb{C}\right)=0$. But this contradicts our assumption on the Hodge type of $(X, x)$, so $D_{J}$ is indeed irreducible.

LEMMA 1.8. Assume that $(X, x)$ is purely elliptic of dimension $n$. Then

$$
h^{0}\left(\Omega_{Y}^{n-1}(\log D) \otimes \mathcal{O}_{D}\right) \leqslant 1
$$

with equality if and only if $(X, x)$ is of Hodge type $(0, n-1)$.

Proof. By (1.5.2), $h^{0}\left(\Omega_{Y}^{n-1}(\log D) \otimes \mathcal{O}_{D}\right)$ is equal to $\operatorname{dim} \operatorname{Gr}_{F}^{n-1} H^{n-1}(U, \mathbb{C})$, hence by semipurity to $h^{n-1,0}=h^{0, n-1}$. The result is now obvious from the definition of Hodge type and the discussion prior to it.

1.9. Examples of purely elliptic singularities. As we shall be mostly concerned with purely elliptic $n$-dimensional $(n \geqslant 2)$ singularities of Hodge type $(0, n-1)$, let us give a brief recipe of how to produce examples.

Let $f: \mathbb{C}^{n+t} \rightarrow \mathbb{C}^{t}$ be a polynomial map whose vanishing defines the germ $(X, 0)$ of an ICIS at 0 . Assume that $f$ is weighted homogeneous with respect to the standard coordinates $x_{1}, \ldots, x_{n+t}$ (resp. $y_{1}, \ldots, y_{t}$ ) of strictly positive integral weights $w_{1}, \ldots, w_{n+t}$ (resp. $d_{1}, \ldots, d_{t}$ ) in the source (resp. target). Then $\omega(1.3 .1)$ may be chosen weighted homogeneously as

$$
\omega=\frac{d x_{1} \wedge \ldots \wedge d x_{n+t}}{d f_{1} \wedge \ldots \wedge d f_{t}}
$$

Thus, $\omega$ is of weight $Q=\left(w_{1}+\cdots+w_{n+t}\right)-\left(d_{1}+\cdots+d_{t}\right)$. Let $B \stackrel{\pi}{\rightarrow} \mathbb{C}^{n+t}$ be the weighted blow-up of the source. Then the strict transform $Y$ of $X$ is a V-manifold, i.e. has only (cyclic) quotient singularities, and the exceptional divisor $D \subset Y$ has V-normal crossings. From [St2, 2.6 and Cor. 3.4] it is not hard to see that the invariants $H^{n-1}\left(\mathcal{O}_{Y}\right)$ and $H^{n-1}\left(\mathcal{O}_{Y}(-D)\right)$ of 1.1 can be computed from this situation (that is, we don't need a full resolution; a V-resolution suffices). An easy computation now shows that $(X, 0)$ is rational (resp. Du Bois) if and only if $Q>0$ (resp. $Q \geqslant 0)$, thus $(X, 0)$ is purely elliptic precisely when $Q=0$. In this case, $(X, 0)$ is of Hodge type $(0, n-1)$ since $D$ is a complete $V$-manifold.

1.10. Now define a new map-germ $f^{\prime}=f+g:\left(\mathbb{C}^{n+t}, 0\right) \rightarrow\left(\mathbb{C}^{t}, 0\right)$ by adding to $f$ a holomorphic map $g=\left(g_{1}, \ldots, g_{t}\right)$ for which all monomials in $g_{i}$ are of weight $>d_{i}$ for all $i=1, \ldots, t$. Let $\left(X^{\prime}, 0\right)$ be its vanishing. Then the weighted blow-up $\pi$ from before gives a V-resolution $Y^{\prime} \rightarrow X^{\prime}$ in a neighbourhood of 0 , and the exceptional divisor $D^{\prime} \subset Y^{\prime}$ is isomorphic to $D$ as analytic spaces. It follows from the reasoning above that $\left(X^{\prime}, 0\right)$ is purely elliptic of Hodge type $(0, n-1)$ if $Q=0$.

1.11. From weight considerations in the space $T_{X, 0}^{1}$ of first order deformations of $(X, 0)$ (see, for instance, [PW, Prop. 10.5.16]) it follows that any deformation of a purely elliptic surface singularity of the above type is trivial $\left((X, 0)\right.$ and $\left(X^{\prime}, 0\right)$ 
are isomorphic for all choices of $g$ ) and indeed, a purely elliptic Gorenstein surface singularity is quasi-homogeneous if and only if it is of Hodge type $(0,1)$; see 2.8 . For dimensions $n \geqslant 3$ however, starting with a weighted homogeneous $f$ with $Q=0$, the generic choice of $g$ will produce a purely elliptic $\left(X^{\prime}, 0\right)$ of Hodge type $(0, n-1)$ which is not quasi-homogeneous.

\section{Quasi-Homogeneity of Purely Elliptic ICISs}

2.1. Throughout this section $(X, x)$ will be an ICIS of dimension $n \geqslant 2$. Then the Milnor number $\mu$ and the Tjurina number $\tau$ are well-defined. Our main concern is to find conditions that ensure quasi-homogeneity in the purely elliptic case. By 1.9, a necessary condition for $(X, x)$ to be quasi-homogeneous is that it is of Hodge type $(0, n-1)$, but by 1.11 this is generally not sufficient. We shall see that the extra condition needed is that the natural map

$$
\beta: H^{0}\left(\Omega_{Y}^{n-1}(\log D)\right) \rightarrow H^{0}\left(\Omega_{Y}^{n-1}(\log D) \otimes \mathcal{O}_{D}\right)=\operatorname{Gr}_{F}^{n-1} H^{n-1}(U, \mathbb{C})
$$

be surjective. (Here and in the following, we apply the definitions in 1.1.)

This may be motivated as follows. It is a well-known fact that $(n-1)$-cohomology of $U$ may be identified with the $(n-1)$-hypercohomology $\mathbb{H}^{n-1}\left(\Omega_{Y}(\log D)\right)$ of the complex $\Omega_{Y}(\log D)$, and that the filtration bete would compute the Hodge filtration if $Y$ were complete. Now that $Y$ is not complete, the filtration bete is affected by the analytic behaviour of $Y$ 'at infinity' and will not in general give the Hodge filtration; but it does when $(X, x)$ quasi-homogeneous [V, Thm. 3.3], and $\beta$ will then be surjective.

2.2. We shall make use of the following formula due to E. Looijenga and $\mathbf{J}$. Steenbrink [LS], valid (as most of what we have to say in 2.2-2.4) for any ICIS of dimension at least 2 ,

$$
\mu-\tau=\sum_{i=0}^{n-2} h^{i, 0}+a_{1}+a_{2}+a_{3} .
$$

The $h^{i, j}$ were defined in (1.5.1), while the $a_{i}$ are non-negative integers defined relative to a choice of good resolution. It is not very instructive to include the definitions of the $a_{i}$ here. Most important for our purpose is the fact that $a_{3}$ dominates the dimension of $\operatorname{Coker} \beta$. Hence, the assumption $\mu=\tau$ implies that $\beta$ is surjective and that $\operatorname{Gr}_{F}^{0} H^{n-1}(U, \mathbb{C}$ ) is pure of weight $n-1$ (Thus, in the purely elliptic case $(X, x)$ is of Hodge type $(0, n-1))$.

2.3. But we can be more specific. From [V, Lemma 2.7] we get the formula

$$
a_{2}+a_{3}=e_{2}^{n, 0}+\operatorname{dim} \operatorname{Coker} \beta .
$$


Here $e_{2}^{n, 0}$ is the dimension of the cokernel of the natural map

$$
H^{0}\left(\Omega_{Y}^{n-1}(\log D)(-D)\right) \stackrel{d}{\rightarrow} H^{0}\left(\Omega_{Y}^{n}\right)
$$

For later use we note that there is a spectral sequence

$$
E_{1}^{p, q}=H^{q}\left(\Omega_{Y}^{p}(\log D)(-D)\right) \Longrightarrow \mathbb{H}^{p+q}\left(\Omega_{Y}(\log D)(-D)\right)=0
$$

with $E_{1}^{p, q}=0$ if $p+q>n$ ([St2, Thm. 2(b)], [V, 2.2, 2.4]). Clearly, $e_{2}^{n, 0}=\operatorname{dim} E_{2}^{n, 0}$.

2.4. Let us define $\chi=\chi(X, x)=\sum_{i=0}^{n-2} h^{i, 0}+\operatorname{dim} \operatorname{Coker} \beta$. It follows from [V, Prop. 4.2] that $\chi$ is an analytic invariant for any ICIS $(X, x)$. Note that the codomain of $\beta$ has dimension $h^{n-1,0}=h^{0, n-1}$ by semipurity, so $\chi=\operatorname{dim} \operatorname{Gr}_{F}^{0} H^{n-1}(U, \mathbb{C})-r k \beta$.

The following lemma is now immediate once we observe (by consulting [LS]) that $a_{1}=0$ when $(X, x)$ is Du Bois.

LEMMA 2.5. Let $(X, x)$ be a purely elliptic ICIS of dimension $n \geqslant 2$. Then

(1) $\mu-\tau=\chi+e_{2}^{n, 0}$.

(2) $\chi=1-r k \beta$.

(3) The only possible values of $\chi$ are 0 and 1 , and $\chi=0$ if and only if $(X, x)$ is of Hodge type $(0, n-1)$ and $\beta$ is surjective.

The Main Theorem is a corollary to the following theorem.

THEOREM 2.6. Let $(X, x)$ be a purely elliptic ICIS of dimension $n \geqslant 2$. Then the following are equivalent

(1) $(X, x)$ is quasi-homogeneous.

(2) $\mu=\tau$.

(3) $\chi=0$.

(4) $(X, x)$ is of Hodge type $(0, n-1)$ and $\beta$ is surjective.

(5) $\beta$ is non-trivial

2.7. As mentioned earlier, (1) $\Longrightarrow(2)$ is due to Greuel [G1, Satz 0.3]. The implications $(2) \Longrightarrow(3) \Longleftrightarrow(4) \Longleftrightarrow(5)$ follow from Lemma 2.5. It remains to give a proof of $(4) \Longrightarrow(1)$. This will be done in $2.9-2.14$ below.

2.8. It is well-known that a purely elliptic surface ICIS (i.e. $n=2$ ) is either a cusp (if of Hodge type $(0,0)$ ) or simply elliptic (if of Hodge type $(0,1)$ ) [Wat, Thm. 3.20]. With Theorem 2.6 we can prove the slightly weaker statement that $(X, x)$ is quasi-homogeneous if and only if it is of Hodge type $(0,1)$. This follows immediately from the formula

$$
\mu-\tau=\chi=h^{0,0}
$$


which holds for any $\mathrm{Du}$ Bois ICIS of dimension 2 since $a_{2}+a_{3}=$ $e_{2}^{2,0}+\operatorname{dim} \operatorname{Coker} \beta=0$. (Indeed, $E_{1}^{0,1}=0$ by the Du Bois property, hence $E_{2}^{2,0}=E_{\infty}^{2,0}=0$ and $E_{1}^{1,1}=E_{\infty}^{1,1}=0$. Since $\operatorname{Coker} \beta$ embeds into the Serre-dual $H_{D}^{1}\left(\Omega_{Y}^{1}(\log D)\right)$ of $E_{1}^{1,1}$, the claim follows.)

2.9. Proof of $(4) \Longrightarrow$ (1). As usual, let $\Theta_{Y}(\log D)=\mathcal{H}$ om $\left(\Omega_{Y}^{1}(\log D), \mathcal{O}_{Y}\right)$ denote the sheaf of analytic vector fields on $D$ with logarithmic vanishing along $D$. By (1.3.1), contraction of $\pi^{*} \omega$ yields an isomorphism

$$
\Theta_{Y}(\log D)\left(D_{I}-D_{J}+D\right) \stackrel{\cong}{\rightarrow} \Omega_{Y}^{n-1}(\log D) .
$$

Assuming condition (4) in Theorem 2.6 (as we do throughout), surjectivity of $\beta$ implies via (2.9.1) that

$$
\begin{aligned}
0 \rightarrow H^{0}\left(\Theta_{Y}(\log D)\left(D_{I}-D_{J}\right)\right) & \rightarrow H^{0}\left(\Theta_{Y}(\log D)\left(D_{I}-D_{J}+D\right)\right) \\
& \rightarrow H^{0}\left(\Theta_{Y}(\log D)\left(D_{I}-D_{J}+D\right) \otimes \mathcal{O}_{D}\right) \rightarrow 0,
\end{aligned}
$$

is exact. Here $H^{0}\left(\Theta_{Y}(\log D)\left(D_{I}-D_{J}+D\right) \otimes \mathcal{O}_{D}\right) \cong \mathbb{C}$ by Lemma 1.8 since $(X, x)$ is of Hodge type $(0, n-1)$.

2.10. By Lemma $1.7, D_{J}$ is an irreducible exceptional component. From [Wahl1, 1.10.2] we then get an injective morphism

$$
\imath: \mathcal{O}_{D_{J}} \hookrightarrow \Theta_{Y}(\log D) \otimes \mathcal{O}_{D_{J}}
$$

defined as follows. If $\left(y_{1}, \ldots, y_{n}\right)$ are local coordinates on $Y$ centered at some point of $D_{J}$ with $y_{1}=0$ a local equation for $D_{J}$, then $\imath$ maps $1 \in \mathcal{O}_{D_{J}}$ to $y_{1} \partial_{y_{1}}$.

It is easily checked that the identity map of $\Theta_{Y}(\log D)$ produces a well-defined morphism

$$
\Theta_{Y}(\log D) \otimes \mathcal{O}_{D_{J}} \rightarrow \Theta_{Y}(\log D) \otimes \mathcal{O}_{D}\left(D+D_{I}-D_{J}\right),
$$

whose composition with $l$ gives a morphism

$$
\phi: \mathcal{O}_{D_{J}} \rightarrow \Theta_{Y}(\log D) \otimes \mathcal{O}_{D}\left(D+D_{I}-D_{J}\right) .
$$

By the local expression $1 \mapsto y_{1} \partial_{y_{1}}$ and the fact that $D_{J}$ is not in the support of $D+D_{I}-D_{J} \geqslant 0, \phi$ must be injective. Hence,

$$
H^{0}\left(\mathcal{O}_{D_{J}}\right) \stackrel{\phi}{\rightarrow} H^{0}\left(\Theta_{Y}(\log D) \otimes \mathcal{O}_{D}\left(D+D_{I}-D_{J}\right)\right)
$$

is an isomorphism of 1-dimensional vector spaces.

2.11. Now choose $\xi_{Y} \in H^{0}\left(\Theta_{Y}(\log D)\left(D_{I}-D_{J}+D\right)\right)$ such that $\xi_{Y}$ projects to $\phi(1)$ in $H^{0}\left(\Theta_{Y}(\log D)\left(D_{I}-D_{J}+D\right) \otimes \mathcal{O}_{D}\right)$ (cf. (2.9.2)). It is quite standard that $H^{0}\left(\Theta_{U}\right)=$ 
$H^{0}\left(\Theta_{X}\right)$, thus $\xi_{Y}$ maps to a vector field $\xi_{X}$ on $X$. This field preserves the maximal ideal $\mathfrak{m}_{X, x}$ of $\mathcal{O}_{X, x}$ and induces a derivation on $\mathfrak{m}_{X, x} / \mathfrak{m}_{X, x}^{2}$.

2.12. We proceed as in [Wahl2, 3.12]. In local coordinates as before, centered at a point of $D_{J}$ which is regular for $D, \xi_{Y}$ is of the form

$$
\xi_{Y}=y_{1} \partial_{y_{1}}+y_{1} \xi_{Y}^{\prime}
$$

for some $\xi_{Y}^{\prime}$ which is logarithmic along $D_{J}$. Hence, $\xi_{Y}^{\prime}$ preserves for all $k>0$ the ideal $\left(y_{1}^{k}\right)$ generated by $y_{1}^{k}$. Let $k$ be the greatest positive integer satisfying $\pi^{*} \mathfrak{m}_{X, x} \subset\left(y_{1}^{k}\right)$ and pick $g \in \mathfrak{m}_{X, x}$ for which $g \circ \pi$ belongs to $\left(y_{1}^{k}\right)$ but not to $\left(y_{1}^{k+1}\right)$. Write $g \circ \pi$ locally as $y_{1}^{k} h$ for some $h$. Then

$$
\xi_{X}(g) \circ \pi=\xi_{Y}\left(y_{1}^{k} h\right)=k y_{1}^{k} h+y_{1}^{k+1} \partial_{y_{1}}(h)+y_{1} \xi_{Y}^{\prime}\left(y_{1}^{k} h\right),
$$

which modulo $\left(y_{1}^{k+1}\right)$ gives $\xi_{X}(g) \circ \pi \equiv k(g \circ \pi)$. Since $\pi^{*} m_{X, x}^{2} \subset\left(y_{1}^{k+1}\right)$, this shows that $\xi_{X}^{p}(g)$ is non-trivial in $\mathfrak{m}_{X, x} / \mathrm{m}_{X, x}^{2}$ for all powers $p$. Thus, $\xi_{X}$ acts non-nilpotently on the Zariski tangent space of $(X, x)$ at $x$.

We can now apply [Sw, Thm. 4.1] to conclude that $(X, x)$ is quasi-homogeneous, except possibly when $(X, x)$ is a hypersurface singularity of multiplicity 2 (note that a positive-dimensional $\mathbb{C}$-analytic ICIS is reduced).

2.13. For the remaining case we must work a bit harder. Assume that $(X, x)$ is the hypersurface defined by an analytic map-germ $f:\left(\mathbb{C}^{n+1}, 0\right) \rightarrow(\mathbb{C}, 0)$ of multiplicity at least 2 . Let $x_{1}, \ldots, x_{n+1}$ be local coordinates on $1^{n+1}$ centered at 0 , and let $J(f)=\left(\frac{\partial f}{\partial x_{1}}, \ldots, \frac{\partial f}{\partial x_{n+1}}\right)$ denote the Jacobian ideal. Since $f$ has an isolated singularity we may choose $k \geqslant 1$ so that $\mathrm{m}^{k} \subset J(f)$, where $\mathrm{m}$ denotes the maximal ideal in the local ring $\mathcal{O}_{n+1,0}$ of the germ $\left(\mathbb{C}^{n+1}, 0\right)$. Put $R=\mathcal{O}_{n+1,0} / \mathrm{m}^{(n+1) k+2}$. Then $\xi_{X}$ from 2.11 lifts to some vector field $\xi_{\mathbb{C}^{n+1}}$ on $\left(\mathbb{C}^{n+1}, 0\right)$ which in turn induces a $\mathbb{C}$-derivation $\xi_{R}$ on $R$. Let $\xi_{R}^{\prime}: R \rightarrow R$ denote the semi-simple part of the operator $\xi_{R}$. Then $\xi_{R}^{\prime}$ is itself a derivation, polynomial in $\xi_{R}$. Clearly $\xi_{R}$ preserves the ideal $(\bar{f}) \subset R$ generated by the projection $\bar{f}$ of $f$, hence so does $\xi_{R}^{\prime}$, and we may choose a unit $\bar{u} \in R$ so that $\bar{u} \bar{f}$ is an eigenvector for $\xi_{R}^{\prime}$ with eigenvalue $\lambda \in \mathbb{C}$, say. Let $u \in \mathcal{O}_{n+1,0}$ denote a lift of $u$, and let $\xi_{\mathbb{C}^{n+1}}^{\prime}$ be a vector field on $\left(\mathbb{C}^{n+1}, 0\right)$ lifting $\xi_{R}^{\prime}$. Then $\xi_{\mathbb{C}^{n+1}}^{\prime}(u f)$ $\lambda u f \in \mathfrak{m}^{(n+1) k+2} \subset \mathfrak{m}^{2} \cdot J(f)^{n+1}$. By the Briançon-Skoda Theorem $(u f)^{n+1} \in J(u f)$, from which $J(f)^{n+1} \subset J(u f)$ (indeed, $J(f)=J\left(u^{-1}(u f)\right)$ is generated by $\left(\partial(u f) / \partial x_{i}\right)$ $+u f\left(\partial \log u^{-1} / \partial x_{i}\right)$ for $\left.i=1, \ldots, n+1\right)$, whence $\xi_{\mathbb{C}^{n+1}}^{\prime}(u f)-\lambda u f \in \mathrm{m}^{2} J(u f)$. It follows that there exists yet another vector field $\xi_{\mathbb{C}^{n+1}}^{\prime \prime}$ on $\left(\mathbb{C}^{n+1}, 0\right)$ satisfying $\xi_{\mathbb{C}^{n+1}}^{\prime \prime}(u f)=\lambda u f$ and agreeing with $\xi_{\mathbb{C}^{n+1}}^{\prime}$ up to order 2 .

By Saito's theorem [Sa], $(X, x)$ is quasi-homogeneous if $\lambda \neq 0$.

2.14. Assume $\lambda=0$. We will show that this leads to a contradiction. Upon replacing $f$ with $u f$ as defining function for $(X, x)$ we may assume $\xi_{C^{n+1}}^{\prime \prime}(f)=0$. Let $\xi_{X}^{\prime \prime}$ denote the induced vector field on $X$. Then by [L, Lemma 9.6], contraction of $\omega(1.3)$ by $\xi_{\mathbb{C}^{n+1}}^{\prime \prime}$ yields an element $\sigma$ in $H^{0}\left(\Omega_{X}^{n-1}\right)$. Obviously $\pi^{*} \sigma \in H^{0}\left(\Omega_{Y}^{n-1}\right)$, but since $\pi$ 
is constant along $D$ and $\Omega_{Y}^{n-1}(\log D)(-D)$ are the forms in $\Omega_{Y}^{n-1}$ whose restriction to each component of $D$ is trivial, then $\pi^{*} \sigma \in H^{0}\left(\Omega_{Y}^{n-1}(\log D)(-D)\right)$. We conclude from (2.9.1) that $\xi_{X}^{\prime \prime}$ lifts to a vector field $\xi_{Y}^{\prime \prime} \in H^{0}\left(\Theta_{Y}(\log D)\left(D_{I}-D_{J}\right)\right)$. Now we may repeat the arguments of 2.11 and 2.12 with $\xi_{Y}-\xi_{Y}^{\prime \prime}$ in place of $\xi_{Y}$ and conclude that $\xi_{X}-\xi_{X}^{\prime \prime}$ acts non-nilpotently on $\mathfrak{m}_{X, x} / \mathrm{m}_{X, x}^{2}$. But this contradicts the fact that the action of $\xi_{X}^{\prime \prime}$ by construction is the semi-simple part of the action of $\xi_{X}$. Thus, $\lambda \neq 0$ as desired.

This concludes the proof of Theorem 2.6

2.15. Remark. Let $(X, x)$ be an isolated hypersurface singularity defined by some $f:\left(\mathbb{C}^{n+1}, 0\right) \rightarrow(\mathbb{C}, 0), n \geqslant 2$, and let $r$ be the rank of the Hessian of $f$. As pointed out by the referee, any such $(X, x)$ with $r \geqslant 2$ is rational. Thus, purely elliptic hypersurfaces of multiplicity 2 appear only with $r=1$. (And using the procedure in 1.10 it is straightforward to produce examples of such.) In the light of this remark we could avoid 2.13 and 2.14 by refering to the following claim at the end of 2.12 :

Assume $(X, x)$ is an isolated hypersurface singularity with $r=1$. If there is a derivation on $(X, x)$ acting non-nilpotently on $\mathrm{m}_{X, x} / \mathrm{m}_{X, x}^{2}$, then $(X, x)$ is quasi-homogeneous.

This claim extends Theorem 4.1 of [SW] and can be proved using this theorem together with Saito's theorem [Sa]. We omit the details.

2.16. Vector fields on a purely elliptic ICIS. We end with the following description of the vector fields on a purely elliptic ICIS $(X, x)$. This should be contrasted with the equivariance of the minimal good resolution of a surface singularity [Wahl1].

PROPOSITION 2.17. If $(X, x)$ is a Du Bois ICIS, then

$$
H^{0}\left(\Theta_{X}\right)=H^{0}\left(\Theta_{Y}(\log D)\left(D_{I}-D_{J}+D\right)\right) .
$$

In particular, any vector field on a purely elliptic $(X, x)$ lifts to a meromorphic vector field on $Y$ with logarithmic vanishing along the essential divisor $D_{J}$.

Proof. The terms $E_{1}^{p, q}$ of (2.3.3) vanish for $p+q>n$, and $E_{1}^{0, n-1}=0$ since $(X, x)$ is Du Bois. It follows that $E_{1}^{1, n-1}=E_{\infty}^{1, n-1}=0$. By Serre-duality, $H_{D}^{1}\left(\Omega_{Y}^{n-1}(\log D)\right)=0$ so $H^{0}\left(\Omega_{Y}^{n-1}(\log D)\right) \rightarrow H^{0}\left(\Omega_{U}^{n-1}\right)$ is an isomorphism. By (2.9.1) this translates to

$$
H^{0}\left(\Theta_{Y}(\log D)\left(D_{I}-D_{J}+D\right)\right)=H^{0}\left(\Theta_{U}\right)=H^{0}\left(\Theta_{X}\right),
$$

as claimed.

\section{Acknowledgement}

The present work is part of a project which was initiated while the author was visiting Utrecht University on a grant from Carlsbergfondet. 


\section{References}

[G1] Greuel, G.-M.: Dualität in der lokalen Kohomologie isolierter Singularitäten, Math. Ann. 250 (1980), 157-173.

[G2] Greuel, G.-M.: On deformation of curves and a formula of Deligne, In: Algebraic Geometry, Proceedings La Rábida (1981), Lecture Notes in Math., 961, Springer-Verlag, 1982, pp. 141-168.

[GMP] Greuel, G.-M., Martin, B. and Pfister, G.: Numerische Charakterisierung quasihomogener Gorenstein-Kurvensingularitäten, Math. Nachr. 124 (1985), $123-131$.

[HM] Herzog, J. and Martsinkovski, A.: Gluing Cohen-Macaulay modules with applications to quasihomogeneous complete intersections with isolated singularities, Comment. Math. Helv. 68 (1993), 365-384.

[I] Ishii, S.: On isolated Gorenstein singularities, Math. Ann. 270 (1985), 541-554.

[L] Looijenga, E.: Isolated Singular Points on Complete Intersections, London Math. Soc. Lecture Note Ser. 77, Cambridge Univ. Press 1984.

[LS] Looijenga, E. and Steenbrink, J.: Milnor number and Tjurina number of complete intersections, Math. Ann. 271 (1985), 121-124.

[PW] du Plessis, A. and Wall, C. T. C.: The Geometry of Topological Stability, London Math. Soc. Monogr. (NS), Oxford University Press, 1995.

[Sa] Saito, K.: Quasihomogene isolierte Singularitäten von Hyperflächen, Invent. Math. 14 (1971), 123-142.

[SW] Scheia, G. and Wiebe, H.: Über Derivationen in isolierten Singularitäten auf vollständigen Durchschnitten, Math. Ann. 225 (1977), 161-171.

[St1] Steenbrink, J. H. M.: Mixed Hodge structures associated with isolated singularities, Proc. Sympos. Pure Math. 40 (1983), 513-536.

[St2] Steenbrink, J. H. M.: Vanishing theorems for singular spaces, Systèmes différentiels et singularités, Astérisque 130 (1985), 330-341.

[V] Vosegaard, H.: Generalized Tjurina numbers of an isolated complete intersection singularity, European Singularities Network Preprints Archive (1998).

[Wahl1] Wahl, J.: Vanishing theorems for resolutions of surface singularities, Invent. Math., 31 (1975), 17-41.

[Wahl2] Wahl, J.: A characterization of quasi-homogeneous Gorenstein surface singularities, Compositio Math. 55 (1985), 269-288.

[Wal] Waldi, R.: Deformation von Gorenstein-Singularitäten der Kodimension 3, Math. Ann. 242 (1979), 201-208.

[Wat] Watanabe, K.: On plurigenera of normal isolated singularities. I, Math. Ann. 250 (1980), 65-94.

[XY] Xu, Y.-J. and Yau, S.-T.: Micro-local characterization of quasi-homogeneous singularities, Amer. J. Math. 118(2) (1996), 389-399.

Note added in proof. Recently the author has proved the main theorem also for complete intersections which are not purely elliptic. To appear. 